\section{Avaliação econômica do uso de dexrazoxano na profilaxia de cardiotoxicidade em crianças em tratamento quimioterápico com antraciclinas}

\author{
Economic assessment of dexrazoxane in \\ prophylaxis of cardiotoxicity in children \\ undergoing chemotherapy with anthracyclines
Evaluación económica del uso del dexrazoxano en la profilaxia de cardiotoxicidad en niños en tratamiento quimioterápico con antraciclinas

Ricardo Ribeiro Alves Fernandes 1

Cid Manso de Mello Vianna 2

Paulo Gomes de Freitas 3

Renata Leborato Guerra 1

Flávia Miranda Corrêa 1

doi: 10.1590/0102-311X00191518

\section{Resumo}

O câncer em indivíduos de 0 a 19 anos é considerado raro, quando comparado à incidência em faixas etárias maiores, sendo estimado entre $2 \%$ e $3 \%$ de todos os tumores malignos registrados no Brasil. O uso de antraciclinas está frequentemente associado ao aparecimento de cardiotoxicidade e faz parte de aproximadamente $60 \%$ dos protocolos terapêuticos em oncologia pediátrica. Dentre as estratégias existentes para a prevenção de cardiotoxicidade o dexrazoxano obteve resultados favoráveis pautados em desfechos intermediários (marcadores bioquímicos e medidas ecocardiográficas). Foi desenvolvida, neste trabalho, uma avaliação de custo-efetividade que compare o uso do dexrazoxano em diferentes populações, além de uma avaliação do impacto orçamentário causado pela possível incorporação da tecnologia. Foi utilizado o horizonte temporal de toda a vida do paciente e a perspectiva de análise do Sistema Único de Saúde. Uma análise de impacto orçamentário para cada tecnologia também foi construída. Após uma busca na literatura, foi desenvolvido um modelo de Markov capaz de comparar o uso do dexrazoxano em seis perfis de pacientes com risco de desenvolver cardiotoxicidade. Usar o medicamento nas crianças menores de cinco anos de idade se mostrou a alternativa mais custo-efetiva (razão de custo-efetividade incremental - RCEI de $R \$ 6.156,96)$, seguida de usar em todos os pacientes (RCEI de R\$58.968,70). Caso o preço diminua a um valor menor que $R \$ 250,00$ por frasco, a alternativa de usar em todas as crianças se torna a mais custo-efetiva. $O$ impacto orçamentário ao final de cinco anos foi de $R \$ 30.622 .404,81$ para uso apenas nas crianças menores de cinco anos. Usar a tecnologia em todas as crianças produziria um impacto incremental de R\$ 94.352.898,77.
Correspondência

R. R. A. Fernandes

Rua Raul Pompeia 58, apto. 204, Rio de Janeiro, RJ 22080-002, Brasil.

ricardorafernandes@gmail.com

1 Instituto Nacional de Cancer José Alencar Gomes da Silva, Rio de Janeiro, Brasil.

2 Instituto de Medicina Social, Universidade do Estado do Rio de Janeiro, Rio de Janeiro, Brasil.

3 Ministério da Saúde, Brasília, Brasil. 


\section{Introdução}

No Brasil, as taxas de incidência de câncer pediátrico ajustadas por idade variam de 30,5 a 209,9 por um milhão de habitantes, sendo a maior observada em Goiânia (Goiás) e a menor em Roraima. Entre crianças, a leucemia se apresenta como o câncer mais frequente (26\%) 1 .

A sobrevida estimada para todas as neoplasias entre crianças e adolescentes é de $64 \%$, valor que aumentou significativamente nas últimas décadas. O Instituto Nacional de Câncer José Alencar Gomes da Silva (INCA) estimou, em 2016, a incidência de câncer em crianças de até 18 anos em 12.500 novos casos 1 . A população nessa faixa etária representa $33,1 \%$ do total da população brasileira segundo o Instituto Brasileiro de Geografia e Estatística - IBGE (Censo Demográfico de 2010. https://censo2010.ibge.gov.br/, acessado em 03/Nov/2015).

O tratamento do câncer com medicamentos da classe das antraciclinas está frequentemente associado ao aparecimento de cardiotoxicidade. Dos problemas cardiovasculares relacionados, pode-se listar insuficiência cardíaca como o mais prevalente, somados a hipertensão arterial sistêmica, arritmia, eventos tromboembólicos, isquemias miocárdicas e pericardiopatias 2 . A maioria dos pacientes pediátricos desenvolve cardiotoxicidade tardia. Após seis anos do fim do tratamento, em torno de 65\% dos pacientes possuem anormalidades estruturais ou funcionais detectáveis no ventrículo esquerdo 3 .

As antraciclinas fazem parte de aproximadamente $60 \%$ dos protocolos terapêuticos em oncologia pediátrica 4 . Nesse caso, $65 \%$ das crianças apresentarão disfunção miocárdica subclínica em algum momento de suas vidas, e, no caso da insuficiência cardíaca congestiva, a taxa varia de 2,8 a 10\% 5,6,7. Em média, duas em cada três crianças apresentarão alguma complicação cardíaca, e 40\% delas poderão desenvolver algum desfecho clínico trinta anos ou mais após o término do tratamento, podendo evoluir para óbito ${ }^{6}$. Dentre as crianças que sobrevivem ao câncer, a taxa de mortalidade por complicações cardiovasculares é cerca de dez vezes maior que na população em geral 5 .

Vários fatores de risco relacionados à cardiotoxicidade das antraciclinas já foram elucidados. A associação mais claramente definida é entre a dose de antraciclina e o desenvolvimento de doenças cardíacas. Embora se observe que quanto maior a dose do medicamento maior a probabilidade de ser cardiotóxico, foi observado que nenhuma dose do medicamento se mostrou segura em relação à cardiotoxicidade. Por ter maior percentagem de gordura, meninas têm maior probabilidade de desenvolver o agravo que indivíduos do sexo masculino 3,4,5,8,9. A idade também se mostrou um fator importante a se considerar, sendo crianças menores de quatro anos o grupo mais vulnerável. Estudos também mostram que a associação do tratamento farmacológico com radioterapia é um importante fator de risco para o desenvolvimento de complicações cardíacas posteriores 3,4,5,8,9.

Danos subclínicos causados pela quimioterapia, progressivamente, desenvolvem-se em eventos de relevância clínica. As estratégias de prevenção ou redução desses danos subclínicos se tornam fundamentais no manejo da cardiotoxicidade 5,9 .

Duas estratégias de prevenção envolvendo o manejo na administração das antraciclinas foram estudadas: a diminuição do tempo de infusão do medicamento e a redução da dose cumulativa. Ambos mostraram-se efetivos em adultos, mas não em crianças 3,5,9.

Dentre as estratégias de prevenção primordiais, o uso de dexrazoxano mostrou-se a mais promissora, com estudos em pacientes pediátricos, concluindo que seu uso associado às antraciclinas reduziria o risco de desenvolver cardiotoxicidade, pautado em desfechos intermediários 10. Em adultos, a metanálise, realizada por uma revisão Cochrane 10, estimou que o uso de dexrazoxano reduz em 5,5 vezes o risco de desenvolver insuficiência cardíaca. Desfechos clínicos finalísticos, como esse, não foram avaliados nos estudos com crianças 3,5,9,11,12. O acompanhamento de uma coorte de 206 pacientes pediátricos com leucemia linfoblástica aguda demonstrou que o dexrazoxano preveniu ou reduziu o aumento de troponina $\mathrm{T}$, uma das proteínas do músculo cardíaco, sem alterar a efetividade do tratamento com as antraciclinas. Alguns pacientes foram acompanhados em longo prazo $(\mathrm{n}=134)$, e, ainda assim, foram observadas, por meio de ecocardiograma, evidências de cardioproteção relacionada ao uso de dexrazoxano 13 .

O presente estudo tem como objetivo principal realizar uma avaliação econômica e analisar o impacto orçamentário da utilização do dexrazoxano na prevenção de cardiotoxicidade em pacientes pediátricos em uso de antraciclinas no Sistema Único de Saúde (SUS). Foram montados seis cenários possíveis de incorporação para avaliar o custo-efetividade do medicamento na realidade brasileira. 


\section{Métodos}

Realizou-se uma consulta à Cochrane Library (https://www.cochranelibrary.com/, em 16/Nov/2015) com o objetivo de encontrar uma revisão sistemática que abordasse o uso do dexrazoxano como medida profilática para cardiotoxicidade em crianças com câncer em tratamento com antraciclinas. Foram utilizados os descritores "anthracyclines", "children", "cancer" e "dexrazoxane". Foi encontrada apenas uma revisão sistemática que atendia ao objetivo 10 . O estudo avaliou o uso de diversas medidas profiláticas (incluindo o dexrazoxano) em pacientes oncológicos adultos e pediátricos tratados com antraciclinas.

Uma busca foi também realizada na base Center for Reviews and Dissemination (CRD; https:// www.york.ac.uk/crd/) da Universidade de York (Reino Unido), com o objetivo de encontrar estudos de avaliação econômica que abordaram pacientes pediátricos sobreviventes ao câncer e profilaxias de sequelas ocasionadas pelo tratamento. Utilizaram-se os descritores "cost-effectiveness" e "pediatric cancer" OR "childhood cancer".

Dentro dos critérios apresentados, só foi encontrada uma análise de custo-efetividade, baseada em um modelo de Markov 14, que comparou o protocolo de screening para cardiotoxicidade do Children Oncology Group (COG; Estados Unidos) 15, com diferentes alternativas, para estimar eficácia e custo-efetividade dos diferentes protocolos 14 . Os dados foram extraídos e conjugados com os encontrados na revisão da Cochrane 10 e adaptados à realidade brasileira para estimar o custo-efetividade e o impacto orçamentário da incorporação da profilaxia no SUS.

O comparador base utilizado foi a estratégia de não prevenção da cardiotoxicidade. Para a avaliação econômica, foi utilizado o software TreeAge Pro 2016 (http://www.treeage.com/).

\section{Perspectiva de análise}

A avaliação econômica foi realizada sob a perspectiva do SUS. Não há profilaxia preconizada para evitar cardiotoxicidade em pacientes pediátricos no sistema de saúde, e a perspectiva foi adotada em consonância com os objetivos do estudo. Apenas custos diretos relacionados ao sistema de saúde foram considerados.

\section{Horizonte temporal de análise}

O modelo foi estruturado para acompanhar toda a vida do paciente. As morbidades avaliadas são tardias ao tratamento do câncer e necessitam de um modelo com horizonte temporal longo para serem avaliadas adequadamente. Custos e benefícios foram descontados em $3 \%$, seguindo as diretrizes metodológicas para avaliação econômica do Ministério da Saúde 16, que consideram adequado o desconto menor que $5 \%$ para estratégias de prevenção, pois, nesse caso, o investimento se faz no presente, e o benefício só ocorre no futuro.

\section{Utilidade e qualidade de vida}

Não foram encontradas medidas de qualidade de vida baseadas em preferências relacionadas aos desfechos de insuficiência cardíaca realizadas na população brasileira. Empregaram-se as utilidades relacionadas a quatro níveis de insuficiência cardíaca definidos pela New York Heart Association (Estados Unidos) 17. As estimativas foram feitas em 64 homens e mulheres utilizando o método timetrade off. Utilizaram-se medidas médias para as faixas etárias entre 26-45 anos e 45-65 anos 14,18, e uma extrapolação linear de 15 anos como limite inferior e 85 anos como limite superior. Os escores médios de utilidade dos níveis III e IV de insuficiência cardíaca foram empregados para o estado de transição de insuficiência cardíaca 18. Os escores de outros desfechos cardíacos também foram aproveitados de Wong et al. 14. 


\section{População de interesse}

O estudo de Wong et al. 14 considerou dados de nove estudos de coorte que avaliaram a incidência da doença nas crianças sobreviventes ao câncer. Os valores relativos às incidências dos desfechos embasaram a formação de categorias relativas a idade, dose de antraciclina, sexo e radioterapia na região do tórax. Criaram-se 12 perfis de pacientes, com diferentes características, para harmonizar os valores encontrados nos estudos.

A partir desses perfis, foram definidos seis grupos de pacientes, comparados entre si, para estimar a relação de custo-efetividade entre receber ou não a profilaxia contra cardiotoxicidade. Distinguiram-se os pacientes segundo as seguintes condições: (a) sexo feminino; (b) pacientes que fizeram radioterapia no tórax; (c) pacientes menores de cinco anos de idade; (d) pacientes com dose de antraciclina maior que $300 \mathrm{mg} / \mathrm{m}^{2}$; (e) os três perfis dos 12 que apresentavam condição mais grave; e (f) toda a coorte. Os pacientes foram reunidos nessas categorias com base nos fatores de risco existentes para desenvolver cardiotoxicidade $3,5,9$. As incidências de insuficiência cardíaca e disfunção assintomática foram agrupadas em distribuições beta, de acordo com os seis grupos desenhados a partir dos perfis de Wong et al. 14 .

\section{Custos}

Uma busca da literatura pesquisou estudos de custo da insuficiência cardíaca por paciente/ano no SUS. Um estudo transversal retrospectivo 19 , realizado por meio de pesquisa de prontuário médico por 12 meses no Hospital Universitário Antônio Pedro, Universidade Federal Fluminense (HUAP/ UFF), foi utilizado como base para estimar o custo do seguimento ambulatorial empregando uma abordagem de microcusteio. Os 70 pacientes tinham mais de 18 anos e insuficiência cardíaca em estágio C e D em critério definido pelo American College of Cardiology e American Heart Association (Estados Unidos). Como custos diretos, o estudo avaliou internações hospitalares, exames complementares, medicamentos, honorários profissionais e custos operacionais. Em seguida, por meio do Programa de Estudos Avançados em Administração Hospitalar e de Sistemas de Saúde da Universidade de São Paulo (PROAHSA), estimou-se a composição de custos hospitalares em hospitais públicos de São Paulo, Rio de Janeiro e Minas Gerais.

O valor da consulta ambulatorial considerou honorários do médico coordenador e dos residentes, sendo acrescido o custo operacional do HUAP. À unidade ambulatorial, foi atribuído um custo operacional (overhead) de $12 \%$ do total de custos do hospital. Os honorários basearam-se no valor dos salários dos médicos e no número de horas dedicadas ao ambulatório de insuficiência cardíaca.

O custo da hospitalização por dia considerou prospectivamente o uso de recursos, como medicamentos e exames complementares no cálculo do valor médio da diária hospitalar. À unidade de internação, foi atribuído custo operacional (overhead) de 15,7\% do total de custos do HUAP. A metodologia para as estimativas de custo seguiu o estudo de Araujo et al. 19, cujos resultados foram utilizados no presente estudo.

Os valores das consultas, exames complementares e internações foram reavaliados para valores de 2017, por meio do índice da inflação acumulada no período (IPCA). O custo dos medicamentos foi atualizado segundo o Banco de Preços em Saúde (BPS). O preço do dexrazoxano foi obtido em consulta à tabela da Câmara de Regulação do Mercado de Medicamentos (CMED) de outubro de 2017 (PMVG 0\%).

O número de frascos que cada paciente utilizou depende da sua superfície corporal. Dados antropométricos do IBGE forneceram a mediana da altura e peso para ambos os sexos para cada faixa etária. A partir deles, calculou-se a superfície corporal média dos pacientes por idade. O número de frascos de cada subgrupo populacional foi inserido na forma de distribuição triangular, pois, para esse parâmetro, era necessária uma distribuição que considerasse apenas números inteiros e diferenciasse um valor mais frequente. 


\section{Efetividade}

Foram extraídos os dados relativos à cardioproteção do dexrazoxano da revisão sistemática 10, na qual foi descrito o risco relativo para o desenvolvimento de insuficiência cardíaca ao utilizar o dexrazoxano. Para pacientes com disfunção do ventrículo esquerdo assintomática (DVEA), não foi possível separar a população que desenvolveu insuficiência cardíaca e DVEA. Nesse caso, buscou-se o risco relativo utilizado para os pacientes que desenvolveram DVEA de uma população que possuí ambas as condições clínicas 20. Tal limitação foi trabalhada na análise de sensibilidade.

Os trabalhos encontrados sugerem que a mortalidade entre as crianças sobreviventes tem magnitude 4 a 10 vezes maior que a da população em geral 14,21,22. Dada a incerteza dessa estimativa, foi escolhido um valor intermediário entre o mínimo e o máximo da magnitude da mortalidade encontrada na literatura. Aplicou-se o multiplicador 8 nas taxas de mortalidade do IBGE para os casos de morte proveniente de pacientes com DVEA e insuficiência cardíaca. Para pacientes sem DVEA, foi aplicada a mortalidade geral do IBGE. As variações desses valores foram trabalhadas em análise de sensibilidade.

\section{Estrutura do modelo}

Foi elaborado um modelo de Markov para calcular a razão de custo-efetividade incremental (RCEI) da utilização do dexrazoxano na profilaxia da cardiotoxicidade em pacientes pediátricos em uso de antraciclinas em comparação à não realização da prevenção, sendo os resultados expressos em custo por ano de vida ganho ajustado para qualidade (QALY, do inglês quality-adjusted life year).

O modelo de Markov teve início após o tratamento de câncer. $\mathrm{O}$ paciente seguiu sem alterações, com DVEA, migrando para estágios subsequentes de insuficiência cardíaca e morte (Figura 1). Os estados de transição DVEA, "sem alterações", "sobrevive" e "morte" não possuem custos associados. As correções para o meio do ciclo foram realizadas.

\section{Análise de sensibilidade}

Foi executada uma análise de Monte Carlo com dez mil simulações de segunda ordem, produzindo curvas de aceitabilidade que estimam a probabilidade de o medicamento ser custo-efetivo diante de um limiar de custo-efetividade. Para os cenários nos quais o medicamento não se adaptou ao limiar proposto, foi construído um limiar de preço por meio de uma análise de sensibilidade determinística univariada em que o medicamento seria custo-efetivo para aquele grupo populacional.

A Tabela 1 apresenta os parâmetros do modelo. Por convenção, chamamos o grupo que inclui todas as crianças de (a), o grupo só do sexo feminino de (b), só quem fez radioterapia no tórax de (c), crianças menores de cinco anos de (d), dose de medicamento maior que $300 \mathrm{mg} / \mathrm{m}^{2}$ de (e) e crianças com quadro mais grave de (f).

\section{Impacto orçamentário}

A análise de impacto orçamentário incremental foi realizada sob a perspectiva do SUS em um horizonte de cinco anos. Para a construção do modelo, foi considerado o número de pacientes com idade inferior a 18 anos que realizaram quimioterapia no SUS em 2014 (Departamento de Informática do SUS. http://www2.datasus.gov.br/DATASUS/index.php, acessado em 16/Dez/2015). Estimou-se que $60 \%$ dos pacientes pediátricos, realizando esse procedimento, utilizam um protocolo que contém antraciclinas 4 . Foi incorporada a taxa de crescimento populacional (IBGE) ao longo do horizonte temporal no modelo. A dose de antraciclina usada nos estudos foi em torno de $300 \mathrm{mg} / \mathrm{m}^{2}$ ao longo de um ano, e a de dexrazoxano foi de dez vezes a dose do antineoplásico por aplicação 14.

Ao observar os dados do IBGE referentes à mediana da altura e peso médio da população, conseguiu-se calcular a superfície corporal média por idade dos pacientes e, a partir daí, o número de frascos utilizados. No caso de pacientes menores de cinco anos, como a variação da superfície corporal é menor, utilizamos um dado médio. No impacto orçamentário para a população com maior variação na idade, utilizou-se o número de pacientes que fizeram o procedimento por idade, bem como o 


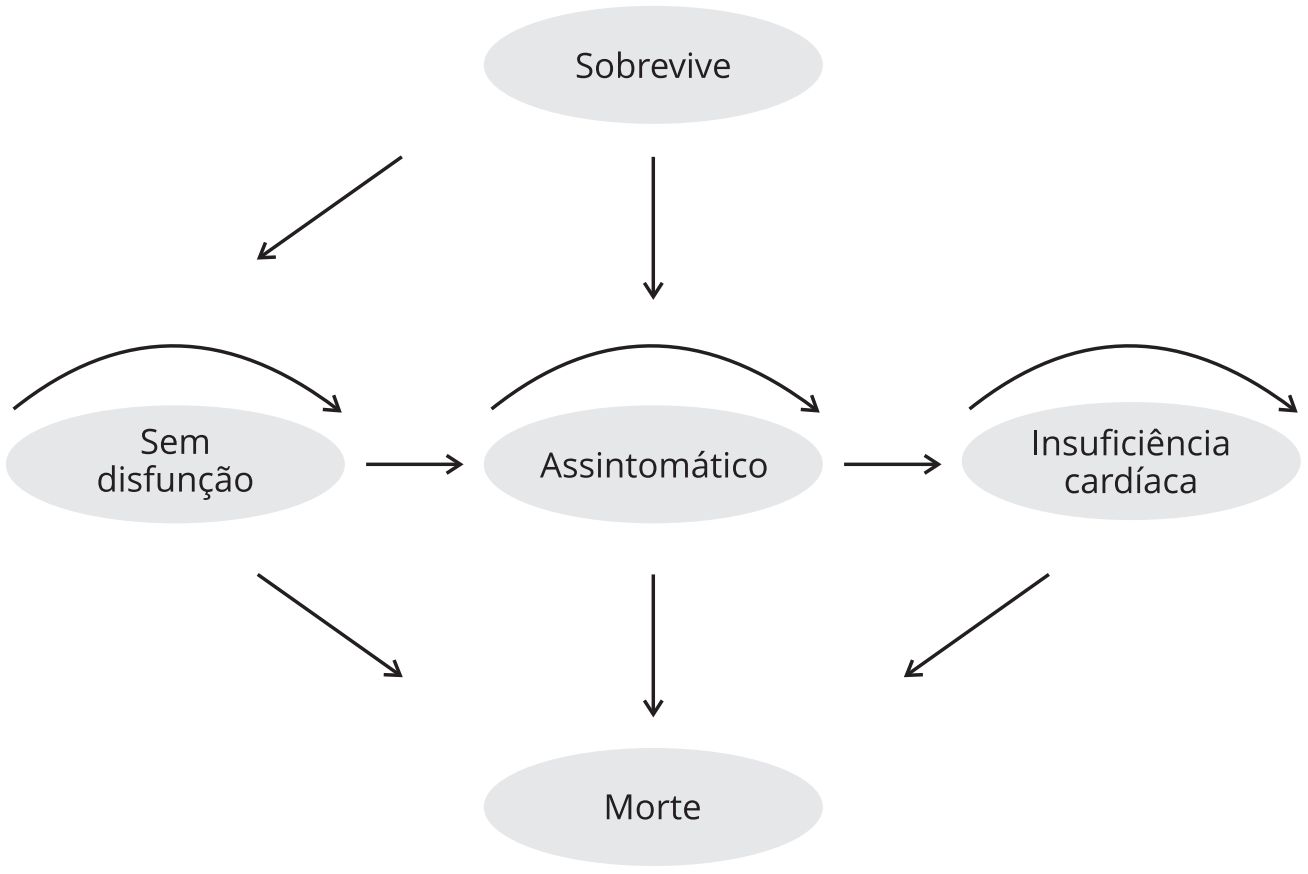

número médio de frascos utilizados segundo a superfície corporal. O impacto orçamentário por ano de incorporação é resultado da multiplicação entre o número de frascos pelo número de pacientes pediátricos em uso de antraciclinas, taxa de crescimento populacional do IBGE e taxa de difusão da tecnologia.

Em consulta à tabela CMED de outubro de 2017, observou-se que o dexrazoxano foi tabelado a R \$ 762,57 por frasco. Como taxa de difusão da tecnologia nos cinco anos do modelo, foram utilizados os valores de 10\%, 30\%, 60\%, 80\% e 100\%, além da taxa de crescimento populacional extraída do IBGE.

\section{Resultados}

Usar o dexrazoxano apenas nas crianças mais graves e nas menores de cinco anos foi a estratégia com maior e menor custo médio, respectivamente ( $\mathrm{R} \$ 7.254,90$ e $\mathrm{R} \$ 3.882,31$ ).

A estratégia que obteve a menor RCEI foi prevenir apenas as crianças menores de cinco anos (R\$ 6.156,96). A estratégia que inclui todas as crianças da coorte (a), se comparada a não tratar, resultou em um RCEI um pouco maior $(\mathrm{R} \$ 7.701,44)$.

Os resultados da simulação estão descritos na Tabela 2, que mostra que o uso da profilaxia apenas em crianças menores de cinco anos de idade é a alternativa mais custo-efetiva, seguida de disponibilizar os medicamentos para todas as crianças.

A estimativa do acúmulo em QALY para a estratégia (d) foi de 15,82, e a estratégia (a) foi a que obteve o maior valor ao fim do horizonte, com 15,84. O valor de RCEI, nesse caso, é estimado em $\mathrm{R} \$ 58.968,70$. A comparação entre o pequeno ganho em QALY da estratégia restritiva de prevenir apenas crianças menores de cinco anos com toda a coorte e uma diferença entre os RCEI de ambas as estratégias comparadas com não tratar $(\mathrm{R} \$ 1.544,48)$ demonstrou pequena diferença entre ambas as estratégias. 
Tabela 1

Custos, efetividades e probabilidades de transição do modelo.

\begin{tabular}{|c|c|c|c|c|c|c|}
\hline Variáveis & Valor médio & DP & Limite inferior & Limite superior & Distribuição & Fonte \\
\hline Probabilidade DVEA (a) & 0,0067 & 0,0030 & NA & NA & Beta & 14 \\
\hline Probabilidade DVEA (b) & 0,0074 & 0,0036 & NA & NA & Beta & 14 \\
\hline Probabilidade DVEA (c) & 0,0080 & 0,0029 & NA & NA & Beta & 14 \\
\hline Probabilidade DVEA (d) & 0,0069 & 0,0030 & NA & NA & Beta & 14 \\
\hline Probabilidade DVEA (e) & 0,0093 & 0,0023 & NA & NA & Beta & 14 \\
\hline Probabilidade DVEA (f) & 0,0119 & 0,0009 & NA & NA & Beta & 14 \\
\hline Probabilidade IC (a) & 0,0041 & 0,0024 & NA & NA & Beta & 14 \\
\hline Probabilidade IC (b) & 0,0045 & 0,0028 & NA & NA & Beta & 14 \\
\hline Probabilidade IC (c) & 0,0054 & 0,0023 & NA & NA & Beta & 14 \\
\hline Probabilidade IC (d) & 0,0041 & 0,0024 & NA & NA & Beta & 14 \\
\hline Probabilidade IC (e) & 0,0061 & 0,0016 & NA & NA & Beta & 14 \\
\hline Probabilidade IC (f) & 0,0080 & 0,0004 & NA & NA & Beta & 14 \\
\hline RR_Morte Cardio & 8 & & 4 & 9 & & 14 \\
\hline RR DVEA & 0,29 & 0,73 & NA & NA & LogNormal & 10 \\
\hline RR IC & 0,18 & 1,18 & NA & NA & LogNormal & 10 \\
\hline Número de frascos $(a, b, c)$ & 6 & NA & 1 & 12 & Triangular & * \\
\hline Número de frascos (d, f) & 5 & NA & 4 & 6 & Triangular & * \\
\hline Número de frascos (e) & 7 & NA & 9 & 12 & Triangular & * \\
\hline Custo IC (R\$) & $11.291,00$ & NA & $9.032,00$ & $13.549,00$ & NA & 19 \\
\hline Preço (R\$) dexrazoxano (frasco) & 762,57 & NA & 610,05 & $1.062,00$ & NA & $\star \star$ \\
\hline $\begin{array}{l}\text { Utilidade DVEA (< } 26 \text { anos; } 26-45 ; \\
46-65 ;>65)\end{array}$ & 0,$96 ; 0,834 ; 0,697 ; 0,51$ & NA & \multicolumn{2}{|c|}{ Não variado } & NA & 18 \\
\hline $\begin{array}{l}\text { Utilidade IC (<26 anos; 26-45; } \\
46-65 ;>65)\end{array}$ & 0,$5 ; 0,427 ; 0,366 ; 0,27$ & NA & 0,$13 ; 0,065 ; 0 ; 0$ & 0,$82 ; 0,79 ; 0,66 ; 0,48$ & NA & 18 \\
\hline
\end{tabular}

DP: desvio padrão; DVEA: disfunção do ventrículo esquerdo assintomática; IC: insuficiência cardíaca; NA: não se aplica; RR: risco relativo; RR_Morte Cardio: risco relativo de morte por eventos cardíacos.

Nota: (a) todas as crianças; (b) grupo só do sexo feminino; (c) só quem fez radioterapia no tórax; (d) crianças menores de cinco anos; (e) dose de medicamento maior que $300 \mathrm{mg} / \mathrm{m}^{2}$; e (f) crianças com quadro mais grave.

* Instituto Brasileiro de Geografia e Estatística - IBGE (Censo Demográfico de 2010. https://censo2010.ibge.gov.br/, acessado em 03/Nov/2015);

** Departamento de Informática do SUS - DATASUS. (http://www2.datasus.gov.br/DATASUS/index.php, acessado em 16/Dez/2015).

\section{Análise de sensibilidade}

Um estudo realizado pelo Instituto de Efectividad Clínica y Sanitaria (IECS; Argentina) 23 propôs limiares de custo-efetividade para países de diferentes perfis de renda, com base no gasto per capita em saúde e na expectativa de vida. Para países com economia similar ao Brasil, os valores situam-se próximo a 1 PIB per capita. O parâmetro que provocou a maior variação no RCEI foi a probabilidade de desenvolver DVEA no grupo sem profilaxia, tornando a alternativa de disponibilizar o medicamento a todas as crianças dominada, quando a variável atinge um valor maior de 0,007. Essa alteração aumenta o RCEI a um patamar quase duas vezes 1 PIB per capita, variando-o de R\$ 2.458,00 a R\$ 52.249,00/ QALY. Tal fato ocorre, pois, o grupo sem profilaxia tem a população mais heterogênea, com as maiores probabilidades de desenvolver as comorbidades por não utilizar a profilaxia. A sensibilidade do preço do frasco pode ser observada quando esse é variado a um valor menor que R\$250,00, e a alternativa de utilizar a tecnologia em todas as crianças se torna a mais custo-efetiva diante do limiar proposto.

A curva de aceitabilidade (Figura 2) mostra uma maior probabilidade de as iterações do grupo (d) serem custo-efetivas desde a faixa dos $\mathrm{R} \$ 10.000,00$ até os $\mathrm{R} \$ 115.000,00$ em que se iguala em probabilidade com o grupo (a). Como a abrangência populacional do grupo (a) é maior, o número de 


\section{Tabela 2}

Custo e efetividade do uso do dexrazoxano em diferentes grupos populacionais.

\begin{tabular}{|c|c|c|c|c|c|}
\hline & Custo (R\$) & Custo incremental (R\$) & $\begin{array}{l}\text { Efetividade } \\
\text { (QALY) }\end{array}$ & $\begin{array}{c}\text { Efetividade } \\
\text { incremental (QALY) }\end{array}$ & RCEI (R\$) \\
\hline \multicolumn{6}{|l|}{ Estratégia } \\
\hline Sem profilaxia & 385,03 & & 15,26 & & \\
\hline Menores de cinco anos de idade & $3.882,31$ & $3.497,28$ & 15,82 & 0,57 & $6.156,96$ \\
\hline Crianças com perfil mais grave & $3.983,92$ & $3.598,88$ & 15,57 & $-0,26$ & Dominado \\
\hline Toda a coorte & $4.891,40$ & $4.506,36$ & 15,84 & 0,02 & $58.968,70$ \\
\hline Sexo feminino & $4.904,57$ & $4.519,54$ & 15,80 & $-0,04$ & Dominado \\
\hline Radioterapia no tórax & $4.926,60$ & $4.541,57$ & 15,77 & $-0,07$ & Dominado \\
\hline Dose de antraciclina $>300 \mathrm{mg} / \mathrm{m}^{2}$ & $7.254,90$ & $6.869,87$ & 15,70 & $-0,14$ & Dominado \\
\hline \multicolumn{6}{|l|}{ Estratégias não dominadas } \\
\hline Sem profilaxia & 385,03 & & 15,26 & & \\
\hline Menores de cinco anos de idade & $3.882,31$ & $3.497,28$ & 15,82 & 0,57 & $6.156,96$ \\
\hline Toda a coorte & $4.891,40$ & $4.506,36$ & 15,84 & 0,02 & $58.968,70$ \\
\hline
\end{tabular}

QALY: ano de vida ganho ajustado para qualidade; RCEI: razão de custo-efetividade incremental.

Fonte: elaboração própria.

\section{Figura 2}

Curvas de aceitabilidade para o uso de dexrazoxano em diferentes grupos de pacientes.

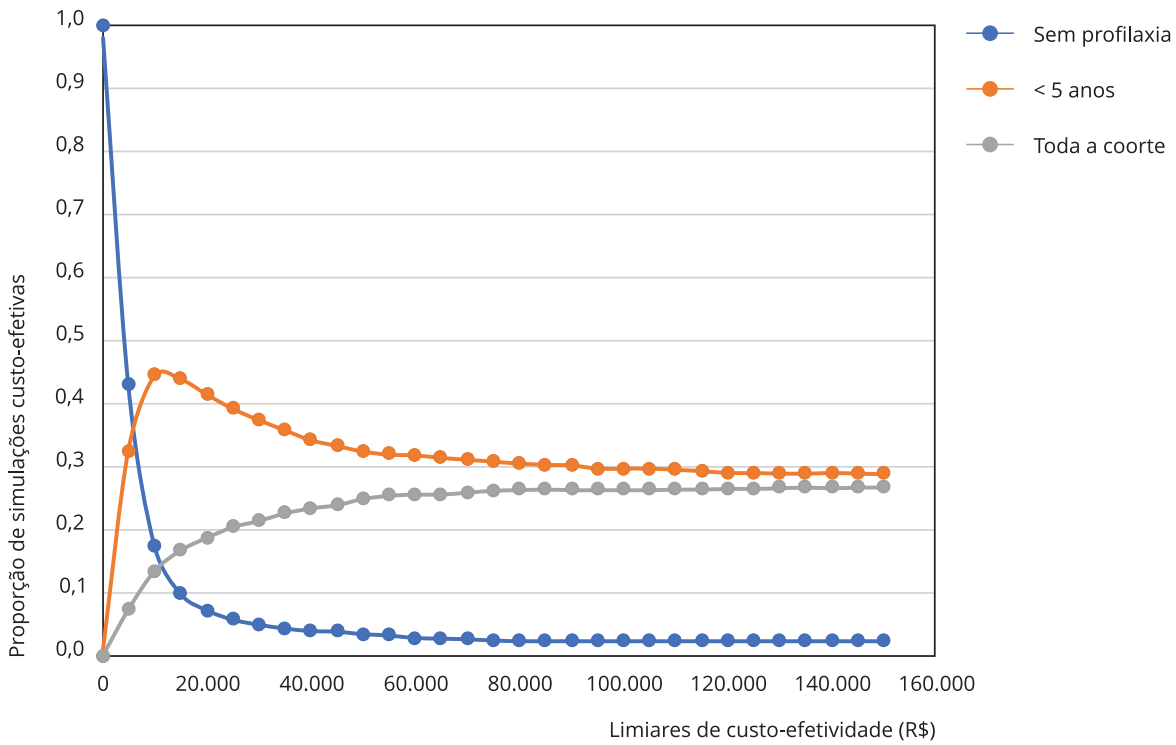

pessoas com maior superfície corporal também é maior, utilizando mais frascos para a intervenção tornando o custo dessa estratégia mais elevado. Com o aumento da disposição a pagar, ela se torna tão custo-efetiva quanto a do grupo (d). No limiar de 1 PIB per capita, a probabilidade de as iterações serem custo-efetivas no grupo (d) é de $37,9 \%$, seguido do grupo (a) $(25,5 \%)$. 


\section{Impacto orçamentário}

Após calcular o número de frascos utilizados segundo os dados de quimioterapia de 2014, observouse, para os pacientes menores de cinco anos, uma média de seis frascos paciente/ano. No caso da população pediátrica total, ao ponderar o número de frascos por faixa etária, estimou-se um número total de frascos anual de 53.818. Foi incorporado ao cálculo o crescimento populacional do IBGE, resultando num total de 58.808 frascos no quinto ano de uso.

Para a população menor de cinco anos, o impacto orçamentário variou de R\$1.005.585,81, no primeiro ano, a R\$11.317.956,86, no quinto ano. Para estratégia de incorporação para todos os pacientes pediátricos, o impacto variou de $\mathrm{R} \$ 4.103 .968,72$, no primeiro ano, a $\mathrm{R} \$ 46.190 .529,54$, no quinto ano. Incorporar a tecnologia para todas as crianças produziria um impacto incremental em relação a usar apenas em menores de cinco anos de $\mathrm{R} \$ 94.352 .898,77$. Esse resultado corresponde a cinco anos de uso da tecnologia. Os resultados da análise estão resumidos na Tabela 3.

\section{Discussão}

Neste estudo, construiu-se um modelo que avaliou o custo-efetividade de diferentes estratégias de prevenção de cardiotoxicidade causada por antraciclinas em pacientes oncológicos pediátricos. Com base nos valores dos RCEIs encontrados e no preço do medicamento praticado no mercado hoje, bem como um limiar de aceitabilidade de 1 PIB per capita, o uso do dexrazoxano em crianças menores de cinco anos de idade mostrou-se custo-efetivo. Diminuir o preço do medicamento para um valor menor que R\$ 250,00 transformaria o RCEI da estratégia de usar em todos os pacientes a um valor abaixo do limiar estimado.

O uso em todas as crianças obteve melhores resultados em saúde do que a alternativa mais custo-efetiva (15,84 vs. 15,82 QALY). Os elevados custos dessa alternativa, que a impossibilitam ser a mais custo-efetiva no ranking, estão associados com a maior heterogeneidade da população desse grupo que possui uma maior variação da superfície corporal em comparação ao grupo (d). Usar apenas em crianças menores de cinco anos possui um custo menor devido à reduzida utilização de frascos do medicamento. O custo dessa estratégia reduz, e seu RCEI fica mais favorável, superando a estratégia do grupo (a). Tal fato deve ser considerado na tomada de decisão para a melhor estratégia e na disposição a pagar pela tecnologia.

As diretrizes das sociedades europeia e americanas de cardiologia reconhecem a necessidade de monitorar os pacientes oncológicos em risco de desenvolver cardiotoxicidade, porém não expressam recomendação específica sobre uma estratégia que previna esse efeito colateral 24 . A alta prevalência de pacientes em risco de cardiotoxicidade por uso de antraciclinas, a grande diferença temporal entre o tratamento e os efeitos cardíacos clínicos, a morbidade e a mortalidade relacionadas a esses efeitos sugerem que o impacto na saúde e as consequências financeiras relacionados a essa condição ainda são subestimados 24 .

Estudos que avaliaram o dexrazoxano na prevenção da cardiotoxicidade especificamente em pacientes oncológicos pediátricos são desconhecidos 25. Paladio-Hernández \& Martínez-Morales 26 fizeram essa avaliação em pacientes com câncer de mama metastático no México. Os autores escolheram a árvore de decisão como estrutura do modelo para estudar desfechos como necessidade de

Tabela 3

Impacto orçamentário do uso do dexrazoxano em crianças menores de cinco anos e em todas as crianças que usam antraciclinas.

\begin{tabular}{lllllll}
\hline Estratégia & Ano 1 (R\$) & Ano 2 (R\$) & Ano 3 (R\$) & Ano 4 (R\$) & Ano 5 (R\$) \\
\hline \multirow{2}{*}{5 anos } & $1.005 .585,81$ & $3.107 .260,15$ & $6.400 .955,90$ & $8.790 .646,10$ & $11.317 .956,86$ \\
Todas as crianças & $4.103 .968,72$ & $12.681 .263,35$ & $26.123 .402,51$ & $35.876 .139,45$ & $46.190 .529,54$ & $124.975 .303,58$ \\
\hline
\end{tabular}

Fonte: elaboração própria. 
cirurgia cardíaca e perda funcional no coração. Os resultados são de difícil comparação com o presente estudo devido à diferença na população estudada e ao desfecho clínico abordado, que influenciou diretamente o horizonte temporal do modelo e a sua estrutura. $\mathrm{O}$ dexrazoxano foi associado a benefícios clínicos como menos eventos cardíacos e menor incidência de insuficiência cardíaca. O autor conclui que utilizar o medicamento como prevenção da cardiotoxicidade é uma estratégia dominante a não utilizá-lo.

Em outra análise de custo-efetividade, Limat et al. 27 avaliaram o uso do dexrazoxano em pacientes com linfoma não-Hodgkin agressivo em tratamento com o protocolo CHOP (ciclofosfamida, doxorrubicina, vincristina e prednisona). Dentre as similaridades metodológicas, esse trabalho empregou o horizonte temporal de toda a vida dos pacientes, e as probabilidades de transição variavam de acordo com a idade e a dose de doxorrubicina utilizada. A expectativa de vida foi o principal desfecho avaliado. Como resultado, foi encontrado um RCEI de R\$ 27.172,05 e R\$ 61.144,96 (1,00 Euro = 3,92 Reais) para pacientes que fizeram cardioproteção com dexrazoxano diagnosticados com 60 e 40 anos de idade, respectivamente. Apesar de o desfecho analisado ser diferente e a idade dos pacientes ser superior a 19 anos, o estudo concluiu que o uso do dexrazoxano deve ser considerado para prevenir cardiotoxicidade.

Os estudos encontrados na literatura diferem deste trabalho em idade da população, desfecho analisado e estrutura do modelo 26,27. Todos corroboram com os resultados encontrados e recomendam positivamente o uso do dexrazoxano como cardioprotetor em pacientes utilizando doxorrubicina, que já possuem seus efeitos cardiotóxicos comprovados 4,5,9.

O Brasil não possui um limiar de custo-efetividade formalizado. Foi considerada, neste estudo, uma estimativa baseada no gasto per capita em saúde e aumento da expectativa de vida. Para o Brasil, esse valor se situa entre 0,5 e 1 PIB per capita (R\$14.000,00 e R\$28.000,00, respectivamente). O resultado do RCEI para a alternativa que atende apenas as crianças menores de cinco anos se situa abaixo desse valor, apontando que essa indicação de uso do medicamento seria custo-efetiva para o sistema de saúde brasileiro. A alternativa de fornecer o medicamento para toda a população resultou em um RCEI que é próximo ao dobro do valor de limiar proposto quando comparado ao da alternativa restritiva (menores de cinco anos). Esse resultado indica, em termos econômicos, que utilizar o medicamento apenas na população restrita é um investimento mais eficiente.

Apesar de o presente estudo apresentar resultados robustos, o modelo possui algumas limitações. A principal delas são os dados relativos à eficácia do medicamento que foram extraídos de uma população composta na maioria por adultos. Os dados relativos à insuficiência cardíaca foram extraídos da meta-análise que conjugou informações tanto relativas à insuficiência cardíaca quanto à DVEA. A idade mostrou-se um fator de risco importante no desenvolvimento de insuficiência cardíaca. Sendo a população infantil mais vulnerável, pode-se esperar melhores resultados do medicamento 14 . Deve-se considerar o uso dessa estratégia profilática como relevante, pois outras alternativas são escassas 10 . A análise de sensibilidade trabalhou as variáveis pertinentes para averiguar o impacto nos resultados finais do modelo e a magnitude da variação dos riscos relativos, não acarretando em alterações nesses resultados.

Outra limitação são as medidas de qualidade de vida, extraídas de um estudo que trabalhou com uma população adulta 18. Os dados foram categorizados nas faixas etárias de 26 a 45 anos e 46 a 65 anos. A limitação é atenuada pelo fato de a condição clínica se estender e se manifestar ao longo de toda a vida adulta do paciente, justamente a faixa etária coberta pelos dados encontrados 6 . A extrapolação linear dos dados de utilidade foi realizada para o limite inferior e superior de idade dos pacientes, o que balanceia a possível subestimação dos valores. Informações sobre qualidade de vida de pacientes brasileiros não estavam disponíveis na literatura.

Os custos da insuficiência cardíaca também são uma limitação importante no modelo, já que os dados extraídos de estudos foram publicados há mais de dez anos. Essa limitação foi mitigada utilizando o IPCA para atualizar os dados relativos a consultas, exames complementares e internações. Ao analisarem tais variáveis na análise de sensibilidade, os resultados finais do modelo não foram alterados. O modelo utiliza dados relacionados ao custo anual por paciente com insuficiência cardíaca. Nesse caso, estudos de custo que utilizam dados de prontuários produzem estimativas mais fidedignas do que as bases de dados do SUS em que as informações estão centradas nos procedimentos. 
O modelo considera que o dexrazoxano não influencia no aparecimento de tumor maligno secundário. Uma meta-análise 28 sugere haver evidência desse evento apenas em pacientes que receberam epipodofilotoxinas ou radiação cranial. O presente estudo não trata dessas especificidades.

Apesar das limitações do modelo, este estudo é o único realizado explorando o potencial custo-efetividade do uso de dexrazoxano como alternativa profilática à cardiotoxicidade em pacientes oncológicos pediátricos brasileiros. Apesar de os dados observacionais retirados do acompanhamento desses pacientes ao longo da vida adulta serem ainda necessários para melhores conclusões, não há informações sobre essa população no Brasil.

\section{Conclusão}

A alternativa de profilaxia mostrou-se custo-efetiva para uma população reduzida (crianças menores de cinco anos). Se excluída a restrição de população na análise, a RCEI da opção de tratar todas as crianças possui um valor pouco diferente $(\mathrm{R} \$ 1.544,48)$ da alternativa que inclui apenas as crianças com idade inferior a cinco anos, assim como o QALY incremental (diferença de 0,02). Tais parâmetros, somados à grande diferença no impacto orçamentário ao incorporar para todas as crianças (R\$ 94.352.898,77 ao fim de cinco anos), devem ser considerados na tomada de decisão.

\section{Colaboradores}

R. R. A. Fernandes elaborou o projeto, desenhou o modelo, analisou, interpretou os dados e redigiu o manuscrito. C. M. M. Vianna orientou o estudo, fazendo parte de todas as etapas de elaboração do modelo econômico, da análise e interpretação dos dados e da revisão final do texto. P. G. Freitas colaborou no desenho do impacto orçamentário, na análise dos dados e na revisão final do artigo. R. L. Guerra e F. M. Corrêa participaram da revisão final do artigo.

\section{Informações adicionais}

ORCID: Ricardo Ribeiro Alves Fernandes (00000002-2197-8724); Cid Manso de Mello Vianna (0000-0003-0252-1144); Paulo Gomes de Freitas (0000-0003-3932-4558); Renata Leborato Guerra (0000-0002-7507-6435); Flávia Miranda Corrêa (0000-0002-1105-2397).

\section{Agradecimentos}

Agradeço ao Instituto de Medicina Social, Universidade Estadual do Rio de Janeiro e aos servidores do Departamento de Gestão e Incorporação de Tecnologias em Saúde, Ministério da Saúde: Fabiana Floriano, Gustavo Laine, Ivan Zimmerman e Vania Canuto.

\section{Referências}

1. Ministério da Saúde. Estimativa 2018: incidência de câncer no Brasil. Rio de Janeiro: Ministério da Saúde; 2018.

2. Santos MVC, Paiva MG, Macedo CRDP, Petrilli AS, Azeka E, Jatene IB, et al. I Diretriz Brasileira de Cardio-oncologia Pediátrica da Sociedade Brasileira de Caridologia. Arq Bras Cardiol 2013; 100(5 Suppl 1):1-68.

3. Harake D, Franco VI, Henkel JM, Miller TL, Lipshultz SE. Cardiotoxicity in childhood cancer survivors: strategies for prevention and management. Future Cardiol 2012; 8:647-70.

4. Schlitt A, Jordan K, Vordermark D, Schwamborn J, Langer T, Thomssen C. Cardiotoxicity and oncological treatments. Dtsch Arztebl Int 2014; 111:161-8.

5. Dillenburg RF, Nathan P, Mertens L. Educational paper: decreasing the burden of cardiovascular disease in childhood cancer survivors: an update for the pediatrician. Eur J Pediatr 2013; 172:1149-60.

6. Franco VI, Henkel JM, Miller TL, Lipshultz SE. Cardiovascular effects in childhood cancer survivors treated with anthracyclines. Cardiol Res Pract 2011; 2011:134679.

7. Shankar SM, Marina N, Hudson MM, Hodgson DC, Adams MJ, Landier W, et al. Monitoring for cardiovascular disease in survivors of childhood cancer: report from the Cardiovascular Disease Task Force of the Children's Oncology Group. Pediatrics 2008; 121:e387-96. 
8. Trachtenberg BH, Landy DC, Franco VI, Henkel JM, Pearson EJ, Miller TL, et al. Anthracycline-associated cardiotoxicity in survivors of childhood cancer. Pediatr Cardiol 2011; 32:342-53.

9. Lipshultz SE, Cochran TR, Franco VI, Miller TL. Treatment-related cardiotoxicity in survivors of childhood cancer. Nat Rev Clin Oncol 2013; 10:697-710.

10. van Dalen EC, Caron HN, Dickinson HO, Kremer LC. Cardioprotective interventions for cancer patients receiving anthracyclines. Cochrane Database Syst Rev 2011; (6):CD003917.

11. Lipshultz SE, Franco VI, Miller TL, Colan SD, Sallan SE. Cardiovascular disease in adult survivors of childhood cancer. Annu Rev Med 2015; 66:161-76.

12. Magnano LC, Martínez Cibrian N, Andrade González X, Bosch X. Cardiac complications of chemotherapy: role of prevention. Curr Treat Options Cardiovasc Med 2014; 16:312.

13. Lipshultz SE, Scully RE, Lipsitz SR, Sallan SE, Silverman LB, Miller TL, et al. Assessment of dexrazoxane as a cardioprotectant in doxorubicin-treated children with high-risk acute lymphoblastic leukaemia: long-term follow-up of a prospective, randomised, multicentre trial. Lancet Oncol 2010; 11:950-61.

14. Wong FL, Bhatia S, Landier W, Francisco L, Leisenring W, Hudson MM, et al. Cost-effectiveness of the children's oncology group longterm follow-up screening guidelines for childhood cancer survivors at risk for treatmentrelated heart failure. Ann Intern Med 2014; 160:672-83

15. Landier W, Bhatia S, Eshelman DA, Forte KJ, Sweeney T, Hester AL, et al. Development of risk-based guidelines for pediatric cancer survivors: the Children's Oncology Group LongTerm Follow-Up Guidelines from the Children's Oncology Group Late Effects Committee and Nursing Discipline. J Clin Oncol 2004; 22:4979-90.

16. Ministério da Saúde. Diretrizes metodológicas: estudos de avaliação econômica de tecnologias em saúde. Brasília: Ministério da Saúde; 2009.

17. Dolgin M, Fox AC, Levin RI, editors. Nomenclature and criteria for diagnosis of diseases of the heart and great vessels. New York: Little, Brown; 1994.

18. Kirsch J, McGuire A. Establishing health state valuations for disease specific states: an example from heart disease. Health Econ 2000; 9:149-58.

19. Araujo DV, Tavares LR, Veríssimo R, Ferraz MB, Mesquita ET. Custo da insuficiência cardíaca no Sistema Único de Saúde. Arq Bras Cardiol 2005; 84:422-7.
20. van Dalen EC, Caron HN, Dickinson HO, Kremer LC. Cardioprotective interventions for cancer patients receiving anthracyclines. Cochrane Database Syst Rev 2005; (1):CD003917.

21. Lawless SC, LaQuaglia MP, Wollner N, Meyers PA, Lindsley KL, Ghavimi F, et al. Desmoplastic small round-cell tumor: prolonged progression-free survival with aggressive multimodality therapy. J Clin Oncol 1996; 14:1526-31.

22. Armstrong GT, Liu Q, Yasui Y, Neglia JP, Leisenring W, Robison LL, et al. Late mortality among 5-year survivors of childhood cancer: a summary from the Childhood Cancer Survivor Study. J Clin Oncol 2009; 27:2328-38.

23. Pichon-Riviere A, Augustovski F, Garcia-Marti S. Derivation of cost-effectiveness thresholds based on per capita health expenditures and life expectancy, and country-level estimates for 194 countries. http://www.iecs.org.ar/wpcontent/uploads/IECS-TechDoc-16-PichonRiviere-et-al-CE-Thresholds-June2016-V2. pdf (acessado em 14/Abr/2016).

24. Nolan MT, Plana JC, Thavendiranathan P, Shaw L, Si L, Marwick TH. Cost-effectiveness of strain-targeted cardioprotection for prevention of chemotherapy-induced cardiotoxicity. Int J Cardiol 2016; 212:336-45.

25. Bryant J, Picot J, Baxter L, Levitt G, Sullivan I, Clegg A. Clinical and cost-effectiveness of cardioprotection against the toxic effects of anthracyclines given to children with cancer: a systematic review. Br J Cancer 2007; 96:22630.

26. Paladio-Hernández J, Martínez-Morales J. Cost-effectiveness of cardioprotective effect of dexrazoxane (cardioxane ${ }^{\circledR}$ ) in advanced/ metastatic breast cancer patients treated with anthracycline-based chemotherapy in Mexico. Value Health 2015; 18:A454.

27. Limat S, Demesmay K, Fagnoni P, Voillat L, Bernard Y, Deconinck E, et al. Cost effectiveness of cardioprotective strategies in patients with aggressive non-Hodgkin's lymphoma. Clin Drug Investig 2005; 25:719-29.

28. Shaikh F, Dupuis LL, Alexander S, Gupta A, Mertens L, Nathan PC. Cardioprotection and second malignant neoplasms associated with dexrazoxane in children receiving anthracycline chemotherapy: a systematic review and meta-analysis. J Natl Cancer Inst 2015; 108:djv357. 


\section{Abstract}

Cancer in individuals 0 to 19 years of age is considered rare when compared to incidence in older age brackets, and is estimated at 2\% to $3 \%$ of all malignant tumors recorded in Brazil. The use of anthracyclines is frequently associated with cardiotoxicity, and these drugs are part of approximately $60 \%$ of treatment protocols in pediatric oncology. Among the existing strategies for the prevention of cardiotoxicity, dexrazoxane obtained favorable results based on intermediate outcomes (biochemical markers and echocardiographic parameters). This study was based on a cost-effectiveness assessment comparing the use of dexrazoxane in different populations, besides an assessment of the budget impact from the technology's potential incorporation. The patient's lifetime was used as the timeline, and the analysis was performed from the perspective of the Brazilian Unified National Health System (SUS). A budget impact analysis was also performed for each technology. After a literature search, a Markov model was developed, capable of comparing the use of dexrazoxane in six profiles of patients at risk of developing cardiotoxicity. Use of the drug in children under 5 years of age proved to be the most cost-effective alternative (incremental cost effectiveness ratio - ICER of BRL 6,156.96), followed by use in all patients (ICER of BRL 58,968.70). If the price decreased to less than BRL 250.00 per vial, the alternative of using the drug in all children would become the most cost-effective. The budget impact at 5 years was BRL 30,622,404.81 for use only in children under 5 years of age. Using the technology in all the children could produce an incremental impact of BRL 94,352,898.77.

Cost-Benefit Analysis; Cardiotoxicity; Dexrazoxane

\section{Resumen}

El cáncer en individuos de 0 a 19 años está considerado raro, cuando se compara la incidencia en franjas etarias mayores, estimándose entre $2 \%$ y 3\% de todos los tumores malignos registrados en Brasil. El uso antraciclinas está frecuentemente asociado a la aparición de cardiotoxicidad y forma parte de aproximadamente un $60 \%$ de los protocolos terapéuticos en oncología pediátrica. Entre las estrategias existentes para la prevención de cardiotoxicidad, el dexrazoxano obtuvo resultados favorables pautados en desenlaces intermedios (marcadores bioquimicos y medidas ecocardiográficas). Se desarrolló en este trabajo, una evaluación de costo efectividad que compare el uso del dexrazoxano en diferentes poblaciones, además de una evaluación del impacto presupuestario causado por la posible incorporación de la tecnología. Se utilizó el horizonte temporal de toda la vida del paciente y la perspectiva de análisis del SUS. También se realizó un análisis del impacto presupuestario para cada tecnología. Tras una búsqueda en la literatura, se desarrolló un modelo de Markov capaz de comparar el uso del dexrazoxano en 6 perfiles de pacientes con riesgo de desarrollar cardiotoxicidad. Usar el medicamento en los niños menores de 5 años de edad se mostró la alternativa más costo-efectiva (relación costo-efectividad incremental - RCEI de BRL 6.156,96), seguido de usarlo en todos los pacientes (RCEI de BRL 58.968,7). En caso de que el precio disminuya a un valor inferior a $B R L$ 250,00 por frasco, la alternativa de usarlo en todos los niños se convierte en la más costo-efectiva. El impacto presupuestario tras 5 años fue de BRL 30.622.404,81 para su uso exclusivo en niños menores de 5 años. Usar esta tecnología en todos los niños, tendría un impacto presupuestario incrementándolo hasta los BRL 94.352.898,77.

Análisis Costo-Beneficio; Cardiotoxicidad;

Dexrazoxano
Recebido em 02/Out/2018

Versão final reapresentada em 12/Mar/2019

Aprovado em 22/Mar/2019 